\title{
Magnetic minor hysteresis loops of compressively deformed transition-metal single crystals
}

\author{
Satoru Kobayashi, ${ }^{a}$ Seiki Takahashi, Yasuhiro Kamada, Hiroaki Kikuchi, and \\ Katsuyuki Ara \\ NDE and Science Research Center, Faculty of Engineering, Iwate University, Ueda 4-3-5, \\ Morioka 020-8551, Japan
}

(Presented on 1 November 2005; published online 27 April 2006)

\begin{abstract}
Magnetic minor hysteresis loops of compressively deformed Ni single crystals have been analyzed. We confirmed universal simple relations between magnetic parameters of minor loops, which are independent of dislocation density, crystal orientation, and the symmetry of crystal. The magnetic properties deduced from the relations are more sensitive to dislocations than those of the major hysteresis loop. Present results clearly show the great capability of minor loops for nondestructive evaluation of degradation in ferromagnetic materials. (C) 2006 American Institute of Physics. [DOI: $10.1063 / 1.2176592]$
\end{abstract}

\section{INTRODUCTION}

Magnetic hysteresis loops of ferromagnetic materials have been the subject of extensive works and have been interpreted in view of the motion of magnetic domain walls and the rotation of magnetic moments. ${ }^{1,2}$ Magnetic properties of the major hysteresis loop, such as coercive force $H_{c}$ and remanence $M_{R}$, reflect statistical behavior of domain wall and give information on the internal stress due to dislocations. The internal stress results in metal fatigue and it is useful to apply the magnetic method to nondestructive evaluation for material degradation before the crack initiation.

Recently, we analyzed magnetic minor hysteresis loops of Fe single-crystal and polycrystalline samples and found simple relations between magnetic parameters of minor loops. Magnetic minor-loop coefficients deduced from the relations are independent of magnetic field amplitude of minor loops and are more sensitive to dislocation density than magnetic properties of the major hysteresis loop. ${ }^{3}$ Nevertheless, the generality of this method for other ferromagnetic materials has not been tested yet, although the type of Bloch wall and its motion at low magnetic fields strongly depend on the symmetry of the crystal. ${ }^{1}$

In the present work, we analyze magnetic minor hysteresis loops of compressively deformed Ni single crystals with fcc structure where various types of domain wall $\left(109^{\circ}\right.$ and $71^{\circ}$ types) play an important role for low-field magnetization process in addition to usual $180^{\circ}$ Bloch wall present in $\mathrm{Fe}$ with bec structure.

\section{EXPERIMENTAL PROCEDURE}

Middle-oriented $\mathrm{Ni}$ single crystals were prepared by electron-beam floating-zone melting method. The cylindrical samples were compressively deformed up to $180 \mathrm{MPa}$ with the strain rate of $1.0 \% / \mathrm{min}$. The true stresses applied to each sample were 16, 40, 62, 97, and $180 \mathrm{MPa}$, and the critical shear stress $\sigma_{c}$ was about $17 \mathrm{MPa}$. For magnetic measure-

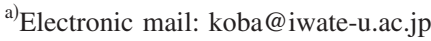

ments, the samples were shaped into a picture frame with (112) surface and the [11ㅣ long axis. The magnetizing and detecting coils with 36 and 50 turns, respectively, were wound on these samples. The magnetic minor hysteresis loops were measured at room temperature, step by step, by changing the magnetic field amplitude $H_{a}$ up to $8 \mathrm{kA} / \mathrm{m}$. To investigate the dependence of minor loops on the crystal orientation, we also prepared some nondeformed samples with (112) surface but with different orientations of the long axis

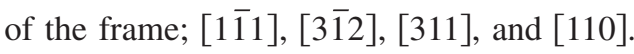

\section{RESULTS AND DISCUSSIONS}

To analyze a set of minor hysteresis loops, we introduce several magnetic parameters of a minor hysteresis loop: minor-loop magnetization $M_{a}^{*}$, minor-loop coercive force $H_{c}^{*}$, minor-loop remanence $M_{R}^{*}$, minor-loop susceptibility at $H_{c}^{*}$, $\chi_{H}^{*}$, minor-loop hysteresis loss $W_{F}^{*}$, and minor-loop remanence work $W_{R}^{*}$ which is the area enclosed by a minor loop in the second quadrant. These parameters correspond to those of the major loop, when $H_{a}$ is large enough to saturate the magnetization.

Figure 1 shows the $H_{a}$ dependence of $M_{a}^{*}$, which corre-

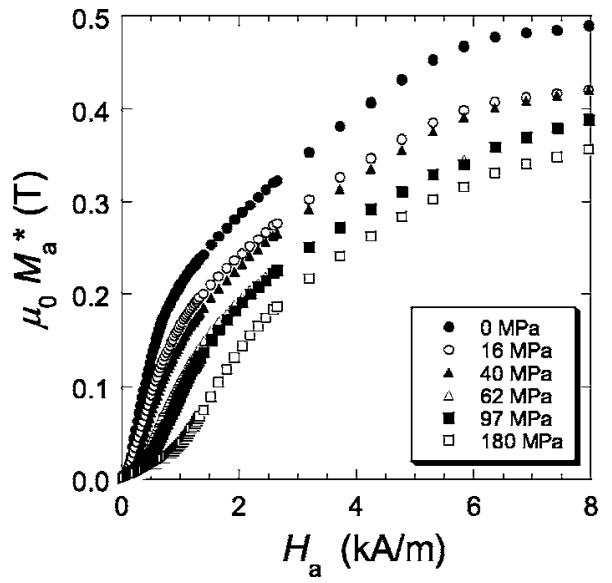

FIG. 1. The dependence of $M_{a}^{*}$ on the magnetic field amplitude $H_{a}$. 

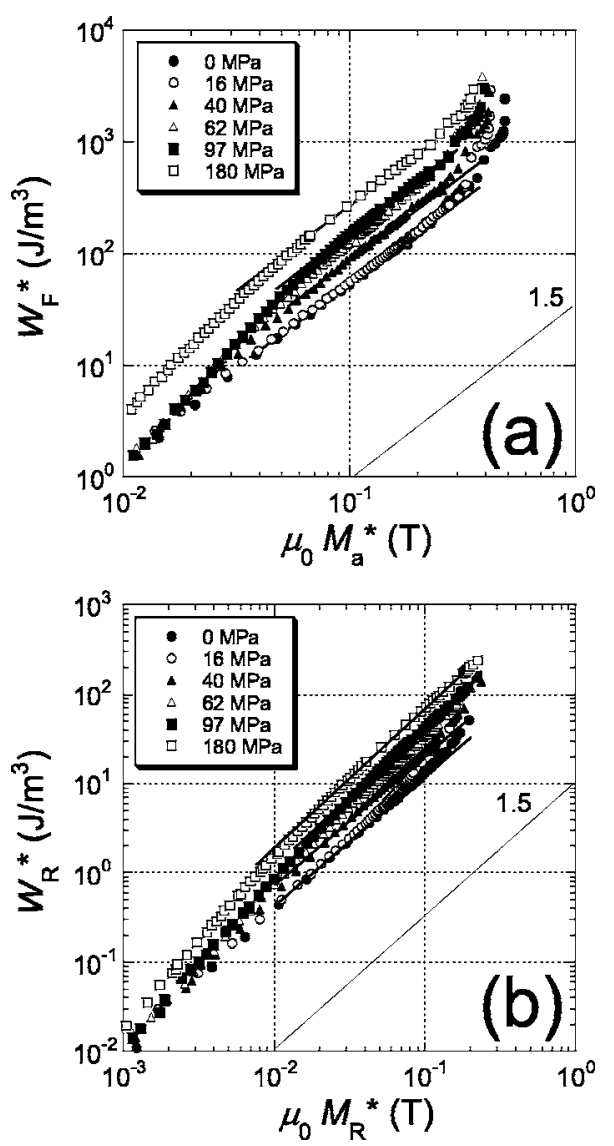

FIG. 2. The relations (a) between $W_{F}^{*}$ and $M_{a}^{*}$ and (b) between $W_{R}^{*}$ and $M_{R}^{*}$. The solid lines denote least-squares fits of the data in the second stage. The slope of 1.5 is shown for comparison.

sponds to the virgin magnetization curve. One can see that $M_{a}^{*}$ changes largely by plastic deformation and decreases systematically at a fixed $H_{a}$ with increasing true stress. Note that the saturation was not achieved even at the maximum field amplitude of $8 \mathrm{kA} / \mathrm{m}$. The magnetization process before the saturation can be divided conveniently into three stages; after a gentle increase of $M_{a}^{*}$ in the first stage, $M_{a}^{*}$ steeply increases with $H_{a}$ in the second stage, followed by a gradual increase of $M_{a}^{*}$ toward the saturation magnetization $M_{s}$ in the third stage. The major part of the magnetization process takes place in the second stage where the movement of Bloch wall plays a crucial role. Since dislocations act as obstacles to the Bloch wall movement and their density increases as the plastic deformation proceeds, the magnetization process would be strongly disturbed by dislocations in the second stage in particular.

All minor-loop parameters depend on the external parameter $H_{a}$ and it is difficult to extract intrinsic physical properties from the $H_{a}$ dependence of minor-loop parameters. It is therefore significant to find another magnetic properties from relations between these minor-loop parameters by taking account of the physical meaning of each minor-loop parameter; $W_{F}^{*}$ is the frictional energy in one cycle of a minor loop and $M_{a}^{*}$ and $M_{R}^{*}$ correspond to the highest position and the trough of the Bloch wall potential, respectively. $W_{R}^{*}$ is the work to displace the Bloch wall from the trough to the crest and corresponds to the depth of the potential. $^{2}$

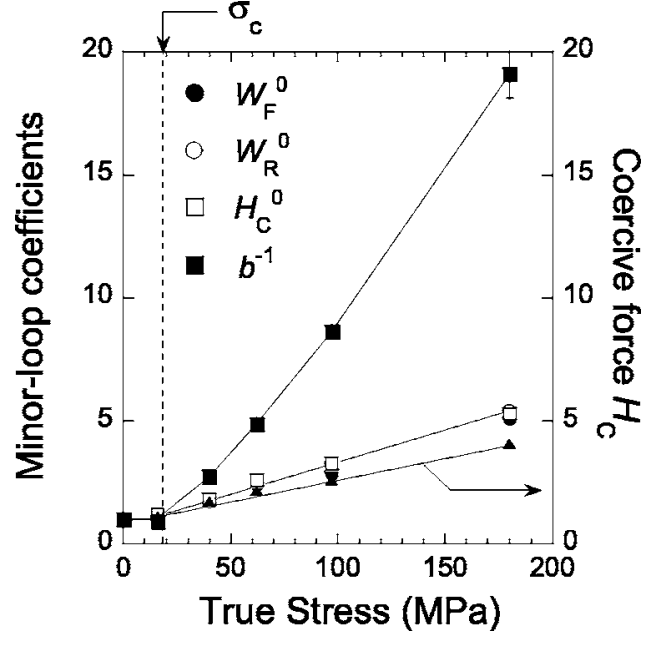

FIG. 3. Minor-loop coefficients, $W_{F}^{0}, W_{R}^{0}, H_{c}^{0}, b^{-1}$, and the coercive force of the major loop, $H_{c}$, as functions of true stress.

In Figs. 2(a) and 2(b), we show double logarithmic plots of relations between $W_{F}^{*}$ and $M_{a}^{*}$ and between $W_{R}^{*}$ and $M_{R}^{*}$, respectively. We found that these relations show a nearly straight line in the second stage and can be fitted, respectively, to a simple equation given by

$$
W_{F}^{*}=W_{F}^{0}\left(\frac{M_{a}^{*}}{M_{s}}\right)^{3 / 2}
$$

and

$$
W_{R}^{*}=W_{R}^{0}\left(\frac{M_{R}^{*}}{M_{R}}\right)^{3 / 2} .
$$

Here, minor-loop coefficients, $W_{F}^{0}$ and $W_{R}^{0}$, are independent of $H_{a}$ and are related to the energy loss and the depth of the Bloch wall potential, respectively. We also found that $H_{c}^{*}$ $-M_{R}^{*}$ and $\chi_{H}^{*}-H_{c}^{*}$ curves show a simple relation in the second stage, given by

$$
H_{c}^{*}=H_{c}^{0}\left(\frac{M_{R}^{*}}{M_{R}}\right)^{1 / 2}
$$

and

$$
\left(\chi_{H}^{*}\right)^{-1}=R_{c}^{0} \exp \left(-b H_{c}^{*}\right),
$$

respectively, where $H_{c}^{0}, R_{c}^{0}$, and $b$ are the minor-loop coefficients. These relations between minor-loop parameters were also obtained for $\mathrm{Fe}$ single crystals deformed plastically. This strongly indicates that the minor-loop relations of Eqs. (1)-(4) are independent of the crystal structure of ferromagnetic materials.

The influence of dislocations on the Bloch wall movement can be quantitatively evaluated using the minor-loop coefficients, and the dependence of the coefficients on true stress is given in Fig. 3. Note that all coefficients are normalized to their value at $0 \mathrm{MPa}$. With increasing true stress, all minor-loop coefficients and $H_{c}$ increase linearly. At each true stress above $\sigma_{c}$, all minor-loop coefficients exceed $H_{c}$; particularly, $b^{-1}$ at $180 \mathrm{MPa}$ is about 20 times larger than that at $0 \mathrm{MPa}$. This indicates that the minor-loop coefficients are very sensitive to dislocations compared with the coercive 


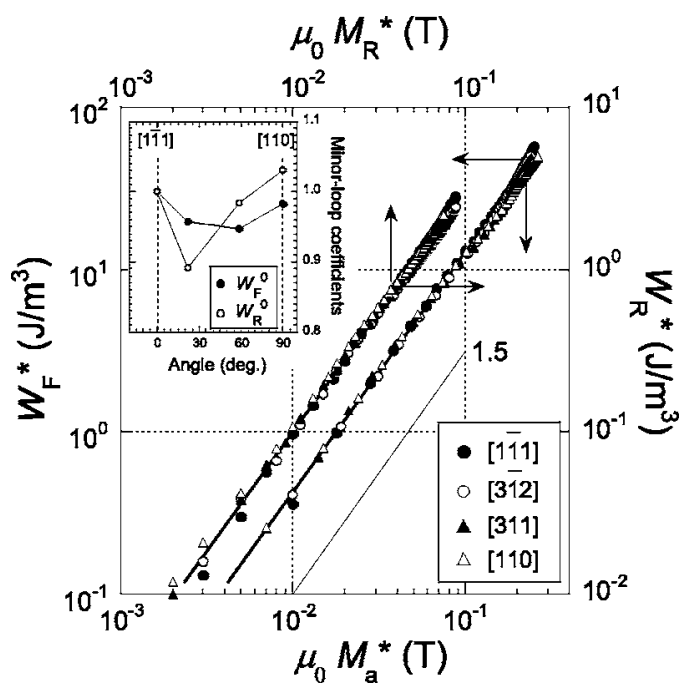

FIG. 4. The relations between $W_{F}^{*}$ and $M_{a}^{*}$ and between $W_{R}^{*}$ and $M_{R}^{*}$ for picture-frame samples with different orientation of the long axis. The inset shows $W_{F}^{0}$ and $W_{R}^{0}$ as functions of the angle between the long axis of the picture frame and the $[1 \overline{1} 1]$ direction.

force of the major loop and that the interaction between dislocations and Bloch wall strongly influences the magnetic susceptibility of minor loops at $H_{c}^{*}$ in particular.

In addition to the stress dependence of the minor loops, we also checked the minor-loop relations for picture-frame samples with different crystal orientations of the long axis. As shown in Fig. $4, W_{F}^{*}-M_{a}^{*}$ and $W_{R^{-}}^{*} M_{R}^{*}$ curves follow the simple relations given by Eqs. (1) and (2), respectively, as was observed for deformed $\mathrm{Ni}$ samples, and the dependence of $W_{F}^{0}$ and $W_{R}^{0}$ on the crystal orientation was obtained. One can see that there exist local maxima of minor-loop coefficients at angles of $0^{\circ}$ and $90^{\circ}$ to the $[1 \overline{1} 1]$ direction as shown in the inset of Fig. 4. This orientation dependence can be explained in view of the magnetic anisotropy energy of $\mathrm{Ni}$ with fcc structure.

Present results show that there exist simple relations between minor-loop parameters that are independent of dislocation density, crystal orientation, and the symmetry of the crystal. This implies the presence of underlying mechanism that governs the motion of the Bloch wall in ferromagnetic materials. The universality, sensitivity, and very low magnetic field to be required for present minor-loop method are very useful characteristics for nondestructive evaluation of degradation in ferromagnetic materials.

\section{ACKNOWLEDGMENTS}

The Ni single crystals were prepared under the interuniversity cooperative research program of the Laboratory for Advanced Materials, Institute for Materials Research, Tohoku University. This research was partly supported by a Grant-in-Aid for Scientific Research (S), Grant No. 14102034, from the Ministry of Education, Culture, Sports, Science, and Technology of Japan.

${ }^{1}$ H. Kronmüler and M. Fähnle, Micromagnetism and the Microstructure of Ferromagnetic Solids (Cambridge University Press, Cambridge, 2003).

${ }^{2} \mathrm{H}$. Träuble, in Magnetism and Metallurgy, edited by A. E. Berkowitz and E. Kneller (Academic, New York, 1969), Chap. 13, p. 621.

${ }^{3}$ S. Takahashi and L. Zhang, J. Phys. Soc. Jpn. 73, 1567 (2004). 